\title{
Prevenção de hemoglobinopatias a partir do estudo em gestantes
}

\author{
Lígia M.S. Viana-Baracioli ${ }^{1,2}$ \\ Cláudia R. Bonini-Domingos ${ }^{1}$ \\ Regina A. Pagliusi \\ Paulo C. Naoum ${ }^{1}$
}

\begin{abstract}
A população brasileira, apresenta genes para as hemoglobinas anormais com freqüências variáveis e influenciadas por seus grupos raciais formadores. Portanto, detecção dos portadores destas alterações genéticas é de importância para a saúde pública pois representam fonte de novos heterozigotos e de possiveis homozigotos. O controle das hemoglobinopatias tem sido possível por meio do aconselhamento genético e diagnóstico precoce. O acompanhamento clínico dos homozigotos, o esclarecimento dos heterozigotos e em especial dos casais de risco, pode contribuir para evitar o nascimento de crianças portadoras de uma patologia genética, muitas vezes letal.

Por essas razões o presente trabalho teve como objetivos: avaliar a importância da análise de gestantes para detecção de hemoglobinopatias com os propósitos de detectar a prevalência e efetuar a prevenção, o estudo familial e a conscientização; para os casos positivos como casal de risco, orientar para o devido encaminhamento médico; avaliar a resposta ao programa.

Do total de 696 gestantes analisadas, 10,7\% apresentaram hemoglobinopatias, com as seguintes prevalências: Talassemia alfa 6, 75\%; Hb AS 2,01\%; Talassemia beta menor 1,29\%; Hb AC 0,28\%; Hb AJ 0,14\%; $\mathrm{Hb}$ AS/Tal. alfa: 0,14\% e P.H.H.F. O,14\%.

A elevada prevalência de hemoglobinopatias encontrada na população de gestantes estudada, evidencia a necessidade da implantação de exames para hemoglobinopatias na rotina de pré-natais, uma vez que neste período as mães estão mais propensas à se preocuparem com sua própria saúde e à de seu bebê e, quanto mais precoce forem diagnosticadas as alterações das hemoglobinas, melhor e mais adequada será a orientação dada ao casal. Rev.bras.hematol.hemoter., 2001, 23 (1): 31-39
\end{abstract}

Palavras-chave: Hemoglobinopatias em gestantes, hemoglobinas anormais, talassemia

\section{Introdução}

A população brasileira caracteriza-se pelo alto grau de mistura racial, com distribuição étnica diferenciada nas várias regiões geográficas do país e em decorrência disso, apresenta prevalência variável de hemoglobinas anormais influenciada por fatores ecológicos e raciais. Síndromes falcêmicas, a hemoglobina $\mathrm{C}$ e as talassemias

Trabalho realizado no Laboratório de Anemias, Departamento de Biologia da UNESP - São José do Rio Preto

1 - Laboratório de Anemias - Departamento de Biologia - UNESP - São José do Rio Preto-SP

2 - Hemocentro de São José do Rio Preto-SP

3 - Laboratório 1 - Instituto Adolfo Lutz - São José do Rio Preto

Correspondência para: Lígia Márcia da Silveira Viana-Baracioli

Rua Moacir Terra Sóssio, 371. CEP: 15170.000. Tanabi. SP

E-mail: bonini@bio.ibilce.unesp.br

Apoio Financeiro: $C N P q$ 
alfa e beta são hemoglobinopatias comuns no Brasil (1, 2, 3, 4, 5, 6) e além disso, tem sido recomendado pela Organização Mundial de Saúde (7), a implantação de programas de prevenção e controle dessas doenças na América Latina e principalmente no Brasil (8).

O sucesso desses programas dependem da receptividade, disponibilidade e interesse da população alvo para este tipo de estudo e por este motivo, a implantação de programas preventivos deste gênero, é muito difícil. A linguagem utilizada na comunicação dos resultados e aconselhamento genético são de fundamental importância, porém, a opinião da pessoa que recebe a informação é decisiva para a eficácia de tais programas, que devem incluir educação populacional, familiar e profissional, aconselhamento genético dos portadores e casais de risco e acompanhamento clínico adequado a cada caso (9). A triagem de hemoglobinopatias é de grande importância quando realizada com base no diagnóstico prénatal, neonatal e em gestantes, para a detecção de portadores assintomáticos, realizando orientação adequada a cada caso, minimizando, assim, os problemas clínicos, psico-sociais e financeiros relacionados à doença $(10,11)$.

Os programas de prevenção para doenças hereditárias destinados a gestantes podem ter boa aceitação, pois, de um modo geral, estas são submetidas ao acompanhamento gestacional, avaliações clínicas e laboratoriais e, portanto, estão mais propensas a cuidar de sua própria saúde e a de seus filhos (12).

Estudos realizados em populações brasileiras revelaram a possibilidade de que existam hoje no Brasil aproximadamente, 10 milhões de pessoas portadoras de hemoglobinas anormais, e que anualmente nasçam cerca de 3 mil pessoas com a forma homozigota $(13,14)$. A organização de um programa preventivo para hemoglobinopatias requer suporte de órgãos oficiais de saúde, diagnóstico realizado por pessoal capacitado e aconselhamento genético de pacientes e casais de risco. O suporte clínico e de puericultura é fundamental para o sucesso de tais programas (15).

Uma vez que a compreensão de uma doença, em especial as de origem hereditária, quando monitoradas no período pré-natal resultam em gravidez normal, com melhor sobrevida dos recém-nascidos, estabelecemos os seguintes objetivos para o presente estudo: detectar a prevalência de hemoglobinas anormais em gestantes, efetuar sua prevenção através do estudo familial, conscientização e aconselhamento genético para São José do Rio Preto e região.

\section{Casuística e Métodos}

O programa proposto foi desenvolvido em doze municípios que fazem parte da Divisão Regional de Saúde de São José do Rio Preto (DIR XXII), estendido para clínicas particulares e instituições públicas de saúde, além da divulgação através de rádio, televisão, jornais e cartazes para que um maior número de gestantes tomassem conhecimento da pesquisa. Foram utilizados para efetuar os testes, $3 \mathrm{ml}$., de sangue colhidos por punção venosa, com o uso de EDTA a 5\% como anticoagulante,com a prévia autorização dos indivíduos envolvidos na pesquisa, segundo orientação do Comitê de Ética em Pesquisa com Humanos. A investigação de hemoglobinopatias foi realizada através de testes seletivos, como: eritrograma (16), análise da morfologia eritrocitária (9), eletroforese em acetato de celulose em pH alcalino (17), teste de resistência osmótica em $\mathrm{NaCl}$ à $0,36 \%$ (18). Os resultados obtidos na triagem, foram confirmados por metodologia específica que incluem: eletroforese em $\mathrm{pH}$ ácido para confirmação de Hb AS e AC (19); dosagens de $\mathrm{Hb}$ A (17) e Hb Fetal (20) para confirmação de Tålassemia Beta; análises citológicas após coloracão vital com Azul Cresil Brilhante (21) nos casos sugestivos de Talassemia Alfa. Nos casos de suspeita de variantes raras, realizamos eletroforese de cadeias polipeptídicas (22).

As fichas das gestantes continham dados de identificação, idade, informações sobre origem racial, idade gestacional, número de filhos, grau de escolaridade e renda familiar. As informações eram posteriormente codificadas para manter o sigilo das participantes. Foram efetuadas para análise dos dados a Estatística descritiva, Teste t de Student e Teste QuiQuadrado (23). 


\section{Resultados}

Do total de 696 gestantes analisadas no período de doze meses, 75 (10,77\%) apresentaram hemoglobinas anormais. Na tabela 01, observamos a relação de cidades participantes do programa, com o número de hemoglobinas anormais encontrado em cada uma delas. Dos doze municípios que participaram do programa, Jales foi o que encaminhou o maior número de amostras de sangue de gestantes, perfazendo $40,53 \%$ do total. Vale ressaltar que a divulgação incluiu centro públicos e particulares de saúde, porém a procura por parte das gestantes atendidas em clínicas particulares foi de apenas 1,4\%.

O tipo de hemoglobinopatia mais freqüênte foi a talassemia do tipo Alfa 47 ( 6,75\%), seguida de falcemia heterozigota com 14 ( 2,02\%) e talassemia Beta heterozigota com nove amostras ( $1,29 \%)$, conforme ilustra tabela 02. Estes três genótipos somados representam 10,06\% do total, e são entre os genótipos encontrados, os passíveis de causarem alterações fisiopatológica quando em homozigose ou combinados entre si.

O retorno dos familiares de gestantes portadoras para estudo familial, foi de $42,2 \%$ por parte dos filhos e de apenas $26,7 \%$ por parte dos companheiros. Entre os companheiros analisados, todos apresentaram hemoglobinas normais. Entre os filhos, cinco $(26,3 \%)$ apresentaram o mesmo tipo de hemoglobina anormal da mãe, sendo três portadores de talassemia Alfa e dois com Hemoglobina AS.

Do total de amostras analisadas, a maioria (79,31\%) foi enviada por outros municípios. Devido a este fato, as informações que deveriam constar da ficha de identificação, principalmente as relativas à origem racial, estavam geralmente incompletos o que não nos permitiu avaliar estatisticamente $100 \%$ das amostras. Por este motivo para efeito de análises estatísticas, foram avaliadas 632 gestantes, sendo 541 caucasóides e 91 negróides com idade média de 23 anos. Destas, 68 eram portadoras de hemoglobinopatias, sendo 48 gestantes caucasóides e 20 negróides. A presença de hemoglobinopatias está relacionada à origem racial, e através do teste qui-quadrado, observamos diferença significativa entre a percentagem de gestantes caucasóides portadoras de hemoglobinas anormais $(8,86 \%$, $\mathrm{n}=48)$ e negróides $(22,22 \%, \mathrm{n}=20)$. O valor de p associado a este caso foi de 0,001.

Entre as portadoras de hemoglobinopatias, verificou-se que, dentre as caucasóides $(n=48)$, $39(7,2 \%)$ eram portadoras de talassemia, sendo $32(5,9 \%)$ do tipo alfa e sete $(1,3 \%)$ do tipo beta; seis $(1,11 \%)$ com Hb AS e três $(0,55 \%)$ eram portadoras de qualquer outra hemoglobina variante $(\mathrm{Hb} \mathrm{AC}, \mathrm{Hb} \mathrm{AJ} e$ interação entre $\mathrm{Hb} \mathrm{AS} /$ Talassemia Alfa). Entre as negróides $(n=20), 11(12,22 \%)$ eram portadoras de talassemia, sendo, $10(11,11 \%)$ do tipo alfa e uma $(1,11 \%)$ do tipo beta; oito $(8,89 \%)$ de $\mathrm{Hb}$ AS e em apenas uma gestante foi encontrada hemoglobina Fetal aumentada com características de Persistência Hereditária de Hemoglobina Fetal (1,11\%). A prevalência de portadoras de talassemia entre as negróides foi elevada, superando o traço falciforme (Hb AS), característico de descendentes de africanos.

Com relação à hematimetria, verificou-se que só houve diferença significativa para a quantidade de glóbulos vermelhos conforme demonstrado na tabela 3, revelando que entre caucasóides evidenciamos uma quantidade média de glóbulos vermelhos superior à existente entre negróides.

No que diz respeito ao grau de escolaridade, a grande maioria (85\%), das gestantes atendidas pelo programa, tinha apenas o Primeiro Grau, sendo este nem sempre completo. Aproximadamente 88\% possuía renda familiar entre um e quatro salários mínimos.

Para as gestantes portadoras de hemoglobinopatia, encaminhamos carta contendo informações sobre o tipo de hemoglobinopatia encontrada, e um convite para que comparecesse ao Centro de Referência de Hemoglobinopatias e/ou Instituto Adolfo Lutz, com seu companheiro e outros filhos. Nesta ocasião, reallizamos aconselhamento genético e coleta de amostra de sangue para exame familial. Quanto aos recém nascidos, as mães foram orientadas para que realizassem pesquisa de hemoglobinopatia após o sexto mês de vida. 
Tabela 1 . Número de gestantes analisadas com hemoglobinas normais e hemoglobinopatias, segundo o local de procedência

\begin{tabular}{l|c|c|c|c|c|c}
\hline Procedência & \multicolumn{2}{|c|}{$\begin{array}{c}\text { Hemoglobinas } \\
\text { normais }\end{array}$} & \multicolumn{2}{c|}{$\begin{array}{c}\text { Com } \\
\text { Hemoglobinopatias }\end{array}$} & \multicolumn{2}{c}{$\begin{array}{c}\text { Total } \\
\text { analisado }\end{array}$} \\
\cline { 2 - 7 } & $\mathbf{n}=$ & $\mathbf{( \% )}$ & $\mathbf{n} \mathbf{0}$ & $\mathbf{( \% )}$ & $\mathbf{n} \mathbf{0}$ & $\mathbf{( \% )}$ \\
\hline Catanduva & 8 & $(1,15)$ & 3 & $(0,43)$ & 11 & $(1,58)$ \\
Dirce Reis & 13 & $(1,87)$ & 1 & $(0,14)$ & 14 & $(2,01)$ \\
\hline José Bonifácio & - & & 1 & $(0,14)$ & 1 & $(0,14)$ \\
Nova Aliança & - & & 1 & $(0,14)$ & 1 & $(0,14)$ \\
Nova Granada & 5 & $(0,72)$ & - & & 5 & $(0,72)$ \\
Jales & 257 & $(36,93)$ & 25 & $(3,59)$ & 282 & $(40,52)$ \\
Pontalinda & 27 & $(3,88)$ & 1 & $(0,14)$ & 28 & $(4,02)$ \\
Santa Albertina & 22 & $(3,16)$ & 7 & $(1,01)$ & 29 & $(4,17)$ \\
S.J.R Preto & 125 & $(17,96)$ & 19 & $(2,73)$ & 144 & $(20,69)$ \\
Tanabi & 91 & $(13,07)$ & 8 & $(1,15)$ & 99 & $(14,22)$ \\
Urania & 66 & $(9,48)$ & 9 & $(1,30)$ & 75 & $(10,78)$ \\
Vitória Brasil & 7 & $(1,01)$ & - & & 7 & $(1,01)$ \\
Total & 621 & $(89,23)$ & 75 & $(10,77)$ & 696 & $(100)$ \\
\hline
\end{tabular}

Tabela 2. Prevalência de hemoglobinopatias entre as 696 gestantes analisadas

\begin{tabular}{c|c|c}
\hline Hemoglobinopatias & $\mathbf{\mathbf { n } ^ { \mathbf { 0 } }}$ & $\mathbf{\%}$ \\
\hline Talassemia alfa & 47 & 6,75 \\
Hb AS & 14 & 2,01 \\
\hline Talassemia beta menor & 9 & 1,29 \\
\hline Hb AC & 2 & 0,28 \\
\hline Hb AJ & 1 & 0,14 \\
\hline Tal.alfa/Hb AS & 1 & 0,14 \\
\hline P.H.H.F. & 1 & 0,14 \\
\hline Total & 75 & 10,47 \\
\hline
\end{tabular}

Tabela 3. Análise estatística para Hematimetria. Comparação segundo a descendência

\begin{tabular}{c|c|c|c|c|c}
\hline Variável & Descendência & $\mathbf{n}^{\mathbf{o}}$ & média & IC (95\%) & valor-p \\
\hline GV milhões $\mathrm{mm}^{3}$ & caucasóide & 48 & 4,25 & 4,$07 ; 4,43$ & $0,0269^{*}$ \\
& negróide & 20 & 3,90 & 3,$70 ; 4,10$ & \\
$\mathrm{Hb} \mathrm{g} / \mathrm{dl}$ & caucasóide & 48 & 11,55 & 11,$19 ; 11,90$ & 0,0928 \\
& negróide & 20 & 10,97 & 10,$36 ; 11,58$ & \\
$\mathrm{Ht} \%$ & caucasóide & 48 & 36,60 & 35,$64 ; 37,56$ & 0,0692 \\
& negróide & 20 & 34,80 & 32,$99 ; 36,60$ & \\
HCM pg. & caucasóide & 48 & 27,48 & 26,$50 ; 28,46$ & 0,8452 \\
& negróide & 20 & 27,65 & 26,$36 ; 28,94$ & \\
\hline
\end{tabular}

*valor significativo ao nível de 5\%. 


\section{Discussão}

No Brasil, a prevalência de hemoglobinas anormais é influenciada por fatores ecológicos e raciais, conforme a região analisada. A estimativa de ocorrência média é de $4 \%$, sem considerar a talassemia alfa, originada por um ou dois genes afetados (2, 9, 24, 25, 26, 27, 28).

Implantar um programa preventivo para hemoglobinopatias em gestantes é importante pois estas já possuem uma rotina de exames prénatal, junto aos quais pode ser anexada a pesquisa de anemia hereditária. Quanto mais precoce o diagnóstico de hemoglobinopatia, principalmente nos casos de homozigoze, o acompanhamento médico adequado poderá ser iniciado, visando melhoria de sobrevida do paciente.

Em nosso estudo ressaltamos o interesse nitidamente diferenciado entre os grupos provenientes de clínicas particulares e centros públicos de saúde. Esperava-se que pessoas com melhor renda familiar, geralmente relacionada a um maior grau de instrução se interessassem mais pela pesquisa, do que pessoas com menor renda familiar e nível de instrução, que se utilizam de centros de saúde. Dois fatores foram importantes para obtenção destes resultados: a) a presença de assistentes sociais e educadoras em centros de saúde, que se empenharam na conscientização sobre o programa das gestantes atendidas; b) a coleta, que foi realizada no próprio centro de saúde, por ocasião da consulta no pré-natal.

Alguns médicos de clínicas particulares revelaram que uma vez orientadas, suas clientes, quando interessadas, procuravam laboratórios particulares para efetuarem os exames e desta forma, não pudemos ter acesso aos resultados obtidos. Desta forma, explicamos o número discrepante entre gestantes atendidas em clínicas particulares ou em Centros Públicos de Saúde. Não realizamos nenhum teste estatístico para verificar se havia diferença significativa ou não nas duas populações estudadas (gestantes atendidas em clínicas particulares e Centros públicos de Saúde), uma vez que esta diferença era muito clara e cálculos com base em uma população de 10 indivíduos comparados a outra de 682 poderiam ficar distorcidos. No entanto em outros estudos no Brasil (12) não houve diferença significativa entre as duas populações estudadas.
Programas para hemoglobinopatias em gestantes realizados em países como os Estados Unidos da América (11) e Brasil (12), mostram resultados satisfatórios. Os programas desenvolvidos no Hemisfério Norte incluem diagnóstico pré-natal do feto, com possíveis intervenções abortivas em seus objetivos, notadamente para casais de risco. Estes objetivos não foram considerados em nossa pesquisa, por não ser aceito pela legislação vigente e apenas procuramos relacionar a probabilidade de nascimento de uma criança homozigota ou heterozigota para hemoglobinopatia visando, conscientização e acompanhamento.

As vantagens e desvantagem de programas pré-natais com objetivos eugênicos para hemoglobinopatias são discutíveis pois além do trabalho de suporte para o diagnóstico fetal, acompanhamento pré e pós aborto, há impedimentos legais e religiosos diferenciados para cada grupo estudado ou país (8).

$O$ índice de heterozigotos para hemoglobinopatias observado entre as gestantes que participaram do nosso trabalho foi de 10,77\%. Estudos realizados no Brasil para detecção de hemoglobinopatias em gestantes revelaram que a prevalência de hemoglobinas anormais foi de 3,6\% (12). A discrepância entre estes valores deve-se ao fato de termos identificado a presença de talassemia alfa por fracionamento eletroforético e avaliação citológica da presença de precipitações intraeritrocitárias de $\mathrm{Hb} \mathrm{H}$ (6,75\%). Outra expectativa nossa, se relacionava às análises dos esposos ou companheiros no sentido de detectar a existência de "casais de risco", condições que podem resultar em nascimento de crianças com formas graves de hemoglobinopatias. Notamos que a adesão por parte do esposo ou companheiro ficou abaixo do esperado, o que pode ser explicado por pelo menos uma das três possibilidades: a) gestantes, morarem separadas dos companheiros, o que dificultaria o contato com o pai; b) o desinteresse, ou o receio de descobrir ser portador de uma alteração genética que até o momento não lhe trouxe problema algum; c) mudança de endereço ou este incompleto, fato que impossibilitou o nosso contato.

A resposta ao programa por parte dos 
esposos encaminhados por serviços públicos, foi significativamente maior, fato justificado por ser o serviço médico que promoveu a coleta de amostras e a conscientização. Nossos resultados demonstram, que níveis de escolaridade e sócioeconômicos mais elevados, não são fatores que favoreçam o interesse por programas preventivos de Hemoglobinopatias. O interesse ou a "sensibilidade" das pessoas envolvidas contam mais no campo genético do que o seu grau de instrução $(8,12)$.

As análises efetuadas possibilitaram detectar 75 portadoras de hemoglobinas anormais, caracterizando um total de sete genótipos, na forma heterozigota: AS, AC, AJ, PHHF, talassemia alfa, talassemia beta e interação entre $\mathrm{Hb} \mathrm{S}$ e talassemia alfa. As $\mathrm{Hb}$ AS e $\mathrm{Hb}$ AC foram as mais freqüentes entre as variantes de hemoglobinas, e a talassemia alfa, a mais prevalente dentre as talassemias, evidenciando que os resultados gerais obtidos apresentam-se similares aos da literatura $(9,12)$.

Dos 45 filhos de gestantes portadoras esperados, 19 (42,2\%) procuraram o nosso serviço para a realização dos exames, específicos sendo que cinco apresentaram o mesmo tipo de hemoglobinopatia da mãe, três com talassemia alfa e dois com Hb AS. Outros estudos (12), relatam a resposta ao programa de 55,3\% dos outros filhos e de 50\% dos recem-natos, número maior do que o encontrado em nossa pesquisa $(0,2 \%)$, provavelmente por ter sido um serviço médico quem realizou o projeto e a coleta das amostras.

Observamos diferença estatisticamente significativa entre portadoras de hemoglobinopatias de acordo com a origem racial. Das 68 gestantes portadoras de hemoglobinopatias, 48 (8,86\%) eram caucasóides e $20(22,22 \%)$ eram negróides, demonstrando a influência da origem racial na prevalência das hemoglobinopatias. Entre as caucasóides houve predomínio de talassemia alfa (5,9\%), provavelmente devido à influência de povos oriundos do Mediterrâneo na formação racial de nossa região. Entre as negróides os tipos de hemoglobinas anormais mais prevalentes foram talassemia alfa $(11,11 \%)$ e traço falciforme $(8,89 \%)$. O esperado seria um número maior de portadoras de traço falciforme, uma vez que a mutação que originou a $\mathrm{Hb} \mathrm{S}$ ocorreu na África, porém em nossa população, é grande a miscigenação racial produzindo variações nas prevalências originais esperadas (1).

Segundo dados da literatura sobre hematimetria na população brasileira, devido à características inerentes à raça, os valores entre negróides encontram-se por volta de 5\% mais baixos, quando comparados com a população caucasóide, estando nossos resultados de acordo com estes dados (29).

Com relação à fisiopatologia das hemoglobinopatias ressaltamos que o heterozigoto falciforme possui em média 40\% de $\mathrm{Hb} \mathrm{S}$ e $60 \%$ de $\mathrm{Hb}$ A, e como esta última tem a capacidade de inibir a polimerização da $\mathrm{Hb} \mathrm{S}$, a morbidade é baixa. Estudos realizados na Jamaica (30) mostraram que não há diferença entre os portadores de hemoglobinas normais quanto à mortalidade e à presença de hematúria, quando comparados com portadores de $\mathrm{Hb}$ AS e que o desenvolvimento somático e sexual também não está comprometido. O comportamento em cirurgias é normal, desde que bem oxigenados. Outros autores, no entanto, acreditam haver maior incidência de hematúria e embolia pulmonar (31). Além disso, há relatos de morte súbita e complicações clínicas em portadores de $\mathrm{Hb}$ AS expostos à condições de baixa tensão de oxigênio, como anestesias prolongadas, esforços físicos extenuantes e trabalhos sob condições adversas (32, 33, 34).

No Brasil, a prevalência da talassemia alfa, caracterizada por alteração de um ou de dois genes alfa, atinge até $12 \%$ da população (13). O portador de talassemia alfa é assintomático, e geralmente a avaliação do eritrograma mostra valores situados no limite inferior de normalidade. No presente trabalho identificou-se talassemia alfa em 11,1\% das gestantes negróides e em 5,9\% das caucasóides. Por outro lado, a talassemia beta menor, ou talassemia beta heterozigota, causa anemia microcítica e hipocrômica de grau leve, com fatigabilidade e dores nas pernas. Em alguns portadores do traço beta talassêmico, a anemia se torna moderada, com presença de cálculo biliar e alterações ósseas (35). No presente estudo observou-se que $1,3 \%$ e $1,1 \%$ das gestantes caucasóides e negróides, respectivamente, eram portadoras de talassemia beta menor ou 
heterozigota. Diante desses resultados, ressaltamos a importância da realização das análises para detecção de talassemias em gestantes, bem como o controle periódico da série vermelha.

\section{Conclusão}

- A quase totalidade das gestantes analisadas nesta pesquisa foi de procedência de centros de saúde, perfazendo 686 das 696 gestantes analisadas. Apenas 10 gestantes foram provenientes de clínicas particulares, apesar da ampla divulgação efetuada sobre o programa para os centros de saúde, clínicas particulares, hospitais, ginecologistas e na mídia.

- Das 696 gestantes analisadas, 75 delas $(10,7 \%)$ apresentaram hemoglobinopatias, com as seguintes prevalências específicas: Talassemia alfa 47 gestantes (6,75\%); Hb AS 14 gestantes (2,01\%); Talassemia beta menor nove gestantes $(1,29 \%)$; $\mathrm{Hb}$ AC duas gestantes (0,28\%); Hb AJ uma gestante $(0,14 \%) ; \mathrm{Hb}$ AS/Talassemia alfa uma gestante $(0,14 \%)$, e P.H.H.F. uma gestante $(0,14 \%)$.

- Dos 75 companheiros das gestantes com hemoglobinopatias, que realizaram as análises para identificação de hemoglobinas anormais, apenas 20 (26,7\%) compareceram. Em nenhum deles detectou-se hemoglobinopatias. Entre os filhos, dos 45 que foram convidados a realizar as análises para identificação de hemoglobinas, somente $19(42,2 \%)$ atenderam ao convite. Desses, cinco apresentaram hemoglobinopatias iguais às da mãe, sendo três portadores de alfa talassemia e dois de $\mathrm{Hb}$ AS.

- A análise da prevalência de hemoglobinopatias por cor da pele e outras características raciais (cabelo e formato do nariz) mostrou que $22,2 \%$ do grupo de gestantes negróides eram portadoras de hemoglobinopatias, enquanto que entre as gestantes de origem caucasóide a prevalência foi de 8,8\%.

- A prevalência dos tipos de hemoglobinopatias entre gestantes, diferenciadas por grupo racial, mostrou que a talassemia alfa foi mais prevalente nos dois grupos. Entre negróides, a prevalência de talassemia alfa foi de 11,1\%, e entre caucasóides de $5,5 \%$. A Hb AS foi mais prevalente no grupo negróide $(8,8 \%)$ que no caucasóide $(1,1 \%)$. A prevalência de talassemia beta menor foi semelhante entre ambos os grupos (1,3\% no caucasóide e 1,1\% no negróide).

Diante dos resultados obtidos, sugerimos que as análises laboratoriais para detecção de hemoglobinopatias façam parte dos exames prénatais. No caso de serem portadoras de anemia hereditária, as gestantes devem ser orientadas sobre o tipo de hemoglobinopatias que a acomete e principalmente no caso de casais de risco, sobre a probabilidade de ter uma criança com a forma homozigota de anemia hereditária.

\section{Hemoglobinopathies: a study in pregnancy}

Lígia M.S. Viana-Baracioli, Cláudia R. BoniniDomingos, Regina A. Pagliusi, Paulo C. Naoum

\section{Summary}

The Brazilian population, presents genes for abnormal bemoglobins with variable frequencies, which are influenced by the founding racial groups. Thus, the detection of carriers of the genetic alterations is important for public bealth, since they represent sources of new heterozygotes and possible homozygotes. The control of the hemoglobin pathologies has been possible by means of genetic counseling and early diagnosis. The clinical follow-up of the bomozygotes and the orientation of the beterozygotes and especially the couples at risk represent a more effective mode of acting to avoid the birth of children who are carriers of a genetic disease, that is frequently lethal.

For these reasons this work had as its objectives: to evaluate the importance of testing in pregnant women for the detection of hemoglobin pathologies with the purpose of investigating the prevalence, attaining prevention, a familial study and awareness; for the positive cases such as couples at risk, orient as to appropriate medical attendance; and to evaluate the response to the program.

Of the total of 696 pregnant women analysed, $10.7 \%$ revealed hemoglobin pathologies with the following rates: alpha Thalassemia 6.75\%; $\mathrm{Hb}$ AS 2.01\%; beta minor Thalassemia 1.29\%; $\mathrm{Hb}$ AC $0.28 \% ; \mathrm{Hb}$ AJ $0.14 \% ; \mathrm{Hb}$ AS/Alpha Thalassemia $0.14 \%$ and P.H.H.F. $0.14 \%$.

The high rates of hemoglobin pathologies encountered in the population of pregnant 
women studied shows the necessity of the implantation of tests for these abnormalities in the pre-natal routine, since in this period the mothers are more apt to be preoccupied with their own health and that of their babies and, however earlier diagnosed the alterations in the hemoglobins, better and more adequate will be the orientations given the couple.

Rev.bras.hematol.hemoter., 2001, 23(1): 31-39

Key words: Hemoglobinopathies in pregnants, abnormal hemoglobin, thalassemia

\section{Referências Bibliográficas}

1. Salzano FM, Tondo CV. Hemoglobin types in Brazilian population. Hemoglobin. 1982. 6:85-97.

2. Naoum PC et al. Hemoglobinas anormais em uma população do Estado de São Paulo, Brasil. Rev. Bras. Patol. Clin., 1983. 19 (3), 86-89.

3. Zago MA, Costa FF. Hereditary haemoglobin desorders in Brazil. Trans. R.Soc.Trop.Med.Hyg., 1985. 79: 385-388.

4. Teixeira RC, Ramalho AS. Genetics and public healt: response of a Brazilian population to a optional hemoglobinopathy program. Rev.Bras.Genet, 1994. 17(4): 435-438.

5. Sonati E et al. Hereditary hemoglobinopathies in a population from Southeast Brazil. Hemoglobin, 1996. 20 (2), 175-179.

6. W.H.O. Memorandum from a W.H.O. meeting. Bull: W.H.O., v. 61, p. 63-80, 1983.

7. Penchszadeh V. Genetics services for hemoglobinopathies in Latin-America. Joint W.H.O. In: Meeting On Prevention And Control Of Hemoglobinopathies, 1993, Nicosia, Cyprius, 3-7

8. Bowman JE. Prenatal screening for hemoglobinopathies. Am.J.Hum.Genet., 1991. 48 (3), 433-438.

9. Domingos CRB. Prevenção das bemoglobinopatias no Brasil: diversidade genética e metodologia laboratorial. 1993. São José do Rio Preto, 1993. 231 p. Tese (Doutorado em Ciências), Instituto de Biociências Letras e Ciências Exatas. Universidade Estadual Paulista.

10. Loader S et al. Prenatal screening for hemoglobinopathies. I. Evaluation of counseling.
Am.J.Hum.Genet., 1991. 48 (3), 447-451.

11. Rowley PT et al. Prenatal screening for hemoglobinopathies. I. A prospective regional trial. Am.J.Hum.Genet., 1991. 48 (3): 439-446.

12. Teixeira RC, Ramalho AS. Genetics and public healt: response of a Brazilian population to a optional hemoglobinopathy program. Rev.Bras.Genet, 1994. 17 (4): 435-438.

13. Naoum, P.C. Hemoglobinopatias e talassemias. São Paulo, Sarvier, 1997. 168p.

14. Cao, A. et al. Thalasssemias in Sardinia: molecular pathology phenothype-genotype correlation and prevention. Am. J. Pediatr. Hem. Onc., 1991. 13(2): 179-188.

15. Naoum, P.C. Diagnóstico das hemoglobinopatias. São Paulo, Sarvier, 1987. 212p.

16. Jannini, P., Jannini-Filho, P. Interpretação Clínica do Hemograma. 10a ed. São Paulo, Sarvier, 1984. 625p.

17. Marengo-Rowe, A.J. Rapid electrophoresis and quantitation of haemoglobin on cellulose acetate. J. Clin. Pathol. 1965. 18: 790-792.

18. Silvestrone, E., Bianco, I. Screening for microcytemia in Italy: analysis of data collected in the past 30 years. Am. J. Hum. Genetic, 1975. 27(2): 198.

19. Vella, F. Acid agar gel electrophoresis of human hemoglobins. Am. J. Clin. Path., 1968. 49: 440.

20. Betke, K., Marti, N.R., Schlicht, I. Estimation of small percentages of foetal haemoglobin. Nature, 1959. 184 (2134): 1877.

21. Papayannopuolos, R., Stamatayannopoulos, G. Stains for inclusions bodies. In: Standartizations of laboratory reagents and methods for detection of haemoglobinopathies. Hew publications, (CDC) Atlanta, Georgia, 1974.

22. Schneider, R.G. Differentiation of electrofhoretically hemoglobins - such as $S$, $D, G$ and $P$ or A2, C, E and $O$ - by electrophoresis of the globin chains. Clinical Chemistry, 1974. 20: 1111-1115.

23. Zar J.H. Biostatistical analysis, $2^{a} \mathrm{ed}$. Prentice Hall, New Jersey, 1984.

24. Salzano, F.M.A. Abnormal hemoglobins studied and counseling in Brazil. In: International Conference Of Sickle Cell Disease: A World Health Problem, 1, 1979, 
Washington: Proceedings of The First International conference of sickle cell disease. Washington: Howard University, 1979.

25. Zago, M.A., et al. Hereditary hemoglobins disorders in a brazilian population. Human. Hered. 1983. 33: 125-129.

26. Naoum, P.C., Mattos, L.C., Cury, P.R. Prevalence and distribution of abnormal hemoglobins in state of Sao Paulo, Brazil. Bull. Pan Am. Health Organ., , 1984. 18: 127-138.

27. Ruiz, M.A., Guerra, C.C.C., Naoum, P.C. Detecção de hemoglobinas anormais em sangue de cordão de recém-nascidos na cidade de Santos, São Paulo, através de eletroforese em gel de agar-amido. Bol. Soc. Bras. Hematol. Hemot., 1986, 7 (137): 8-13.

28. Medeiros, T.M.D., Bezerra, F.L., Gouveia, J.B.B. Tipos de hemoglobinas em escolares da cidade de Natal, RN. Rev. Bras. Anal. Clin. 1992. 24: 79-80.

29. Naoum, P.C. Hemograma. Análise e interpretação. São Paulo, UNESP, 1998. 155p. (Comunicação Pessoal).

30. Ashcroft, M.T., Desai, P. Mortality and mobility in Jamaican adults with sickle-cell trait and with normal hemoglobin followed up for twelve years. Lancet. 1976. 9: 784-786.

31. Britto, M.M.S., Alves, A.F.P., Rabelo, M.M. Alterações do desenvolvimento somático e sexual na anemia falciforme e traço falcêmico. Arq. Bras. Endocrinol. Metabol., 1984. 29(4): 111-114.

32. Beutler, E. The sickle cell diseases and related disorders. In: Hematology 3.ed. New York: Mc Graw Hill, 1983, p.583-609.

33. Hutz M.H., Salzano F.M., Adams J. Hb F levels, longevity of homozygotes and clinical course of sickle cell anemia in Brazil. Am. J. Med. Genet., 1983. 14: 669-676.

34. Charache S. Sudden death in sickle trait. Am. J. Med., 1988. 84: 459-461.

35. Cançado R.D. Talasssemias - manifestações clinicas e tratamento. In: Naoum, P.C. Hemoglobinopatias e talassemias. São Paulo: Sarvier, 1997: 120-136.

Recebido: 07/07/00

Aceito: 20/11/00 